\title{
Joint method using Akamatsu and discrete wavelet transform for image restoration
}

\author{
Jihad Maulana Akbar and De Rosal Ignatius Moses Setiadi \\ Department of Informatics Engineering, Faculty of Computer Science, \\ Dian Nuswantoro University, Semarang, Indonesia
}

\begin{abstract}
Current technology makes it easy for humans to take an image and convert it to digital content, but sometimes there is additional noise in the image so it looks damaged. The damage that often occurs, like blurring and excessive noise in digital images, can certainly affect the meaning and quality of the image. Image restoration is a process used to restore the image to its original state before the image damage occurs. In this research, we proposed an image restoration method by combining Wavelet transformation and Akamatsu transformation. Based on previous research Akamatsu's transformation only works well on blurred images. In order not to focus solely on blurry images, Akamatsu's transformation will be applied based on Wavelet transformations on highlow (HL), low-high (LH), and high-high ( $\mathrm{HH})$ subunits. The result of the proposed method will be comparable with the previous methods. PSNR is used as a measure of image quality restoration. Based on the results the proposed method can improve the quality of the restoration on image noise, such as Gaussian, salt and pepper, and also works well on blurred images. The average increase is around $2 \mathrm{~dB}$ based on the PSNR calculation.
\end{abstract}

Keywords Akamatsu transform, Blurred image, Discrete wavelet transform, Image Denoising,

Image restoration

Paper type Original Article

\section{Introduction}

An image is a discrete representation of data that has layout information and the color intensity of an object [1]. Nowadays, an image can be easily converted into digital content, but sometimes there is additional noise in the image during camera capture so that the image appears damaged. This operation of repairing damage is called image restoration. Damage can occur when shooting can be caused by the quality of the digitizer, poor camera focus, and signal noise from sensors, so that noise or image appears blurry [2]. Image restoration is the process to obtain a clean original image of the damaged image or that exposed to noise [3,4].

(C) Jihad Maulana Akbar and De Rosal Ignatius Moses Setiadi. Published in Applied Computing and Informatics. Published by Emerald Publishing Limited. This article is published under the Creative Commons Attribution (CC BY 4.0) license. Anyone may reproduce, distribute, translate and create derivative works of this article (for both commercial and non-commercial purposes), subject to full attribution to the original publication and authors. The full terms of this license may be seen at http:// creativecommons.org/licences/by/4.0/legalcode

Declaration of Competing Interest: The authors declare that they have no known competing financial interests or personal relationships that could have appeared to influence the work reported in this paper.

Publishers note: The publisher wishes to inform readers that the article "Joint method using Akamatsu and discrete wavelet transform for image restoration" was originally published by the previous publisher of Applied Computing and Informatics and the pagination of this article has been subsequently changed. There has been no change to the content of the article. This change was necessary for the journal to transition from the previous publisher to the new one. The publisher sincerely apologises for any inconvenience caused. To access and cite this article, please use Akbar, J. M., De Rosal., I. M. S. (2019), "Joint method using Akamatsu and discrete wavelet transform for image restoration", Applied Computing and Informatics. Vol. ahead-of-print No. ahead-of-print. https://10.1016/ j.aci.2019.10.002. The original publication date for this paper was 12/10/2019.
Joint method for image restoration

Received 10 June 2019 Revised 5 October 2019 Accepted 9 October 2019 . 
The image restoration has been done with various methods, such as Akamatsu transform [5], Wavelet Transform [6-8], Anscombe transforms [9], Stochastic Resonance [7], Singular Value Decomposition(SVD) [6,10], Adaptive Histogram Equalization [6].

Karungaru et al. [5] used Akamatsu transformation to restore the image. Akamatsu transform is a transformation technique developed by Norio Akamatsu. Akamatsu transform produces a differential value showing the characteristics of the image and by scaling between the integral and differential values can perform image restoration. From this integral and differential values, there is a scale adjustment between the integral and the differential values to obtain the image of the restoration result. The result of restoration with this method is very good only on the blurred image because it can increase the energy in the image, but produce side effects as other noises appear sharper.

Megha et al. [6] proposed Adaptive Histogram Equalization and DWT techniques to restore the image. The first step of image restoration is done by decomposing using Discrete Wavelet Transform to get four low-low (LL), low-high (LH), high-low (HL), and high-high $(\mathrm{HH})$ sub-domains. Sub-band LL is selected then copied to LL2 to be applied to Adaptive Histogram Equalization. Sub-band LL and LL2 subsequently decomposed using SVD to produce U1, S1, V1 and U2, S2, V2. Then the calculation of both SVD decomposition to determine the improvement factor. The results of this restoration method are good for removing noise but making the energy in the image is reduced.

SVD is a transformation that has been widely used in digital image processing [10-12]. SVD is a technique for obtaining geometric features of an image. Basically, SVD is used to handle matrices that do not have an inverse. The SVD has several characteristics that the singular value change does not significantly alter the overall image and the singular value contains illumination information, while the singular vector retains the information from the image.

Wavelet Transform is a technique for stable signals and not stationary. Because of its efficiency in analyzing local discontinuities of a signal, the Wavelet Transform has been widely used in various disciplines [13-16]. DWT is one form of Wavelets Transform in which the image signal is passed through a pile of analysis filters followed by a subtraction operation. DWT decomposes images into 4 sub-domains: low-low (LL), low-high (LH), highlow (HL), and high-high (HH). LL is the low-frequency signal which is the approximation of the original image. $\mathrm{LH}$ and $\mathrm{HL}$ are intermediate frequency signals. And $\mathrm{HH}$ is a highfrequency signal. $\mathrm{LH}, \mathrm{HL}$, and $\mathrm{HH}$ represent changes in information in the image [13,17]. So DWT is very good at representing a signal with singularity. However, DWT is not suitable for representing fine image signals and colored noise so it needs to be combined with other methods to improve image restoration results [18].

Akamatsu transform proved excellent for restoring the image blur due to produce a different value which is characteristic of the image. This value will be strengthened and can reduce the blur in the image. While the use of low sub-band on wavelet transforms can reduce noise but make significant energy changes in the image. Based on this background, this research proposes to combine DWT and Akamatsu to get more optimal results of image restoration. Sub-bands $\mathrm{LH}, \mathrm{HL}$, and $\mathrm{HH}$ were chosen to apply Akamatsu transformation. To test the quality of restoration method will be given various image noise such as Gaussian noise, salt \& pepper, and blur. The result of the restoration will be calculated by the PSNR and compared with the method contained in the research Karungaru et al. [5] and Megha P et al. [6].

\section{Akamatsu and wavelet transform}

\subsection{Akamatsu transform}

Akamatsu transformation is a transformation that produces the integral and differential values of an input signal $[5,19]$. Akamatsu transformation requires a target signal that is 
defined as $P(x, y)$ Where the target signal is in the transformation. With the presence of the target signal, the result of the transformation will be divided into two parts, namely the right of the target signal and the target signal left. The signals to the right of $P(x, y)$ are defined as $\operatorname{VRS}(x)$ and left as $\operatorname{VLS}(x)$. The target signal is used to determine the result of Akamatsu transformation. To see more clearly the description of the target signal on Akamatsu transform can see Figure 1.

To achieve the target signal required the calculation of the integral value and the differential value. To get an Integral value can use Eq. (1).

$$
A(x)=\frac{\left[\operatorname{Avr} S_{R}(x)+\operatorname{Avr} S_{L}(x)\right]}{2}
$$

with $\operatorname{AvrSR}(x)$ and $\operatorname{AvrSL}(x)$ obtained from Eqs. (2) and (3).

$$
\begin{aligned}
& A v r S_{R}(x)=\frac{S_{R}(x)}{N} \\
& \operatorname{Avr}_{L}(x)=\frac{S_{L}(x)}{N}
\end{aligned}
$$

While the first order of the integral value of the Akamatsu transform is defined by Eq. (4).

$$
[A(x)]^{1 s t}=A(x)
$$

Thus, the member values of $\operatorname{VRS}(x)$ and $V L S(x)$ can be written by Eqs. (5) and (6).

$$
\begin{gathered}
\operatorname{VRS}(x)=P(x+1, y), P(x+2, y), . ., P(x+N, y) \\
V L S(x)=P(x-1, y), P(x-2, y), . ., P(x-N, y)
\end{gathered}
$$

$N$ is an optional constant value that can be changed in every order. While the sum of $\operatorname{VRS}(x)$, defined as $S_{R}$ can be calculated by Eq. (7).

$$
S_{R}=\sum_{k=1}^{N} P(x+k)
$$

and the sum of $V L S(x)$, defined as $S_{L}$ which can be calculated by Eq. (8).

$$
S_{L}=\sum_{k=1}^{N} P(x-k)
$$

Having obtained the value of Integral it can be obtained deferential value. The differential value $(D)$ is obtained by calculating the difference of the original pixel value with the integral value found in Eq. (9).

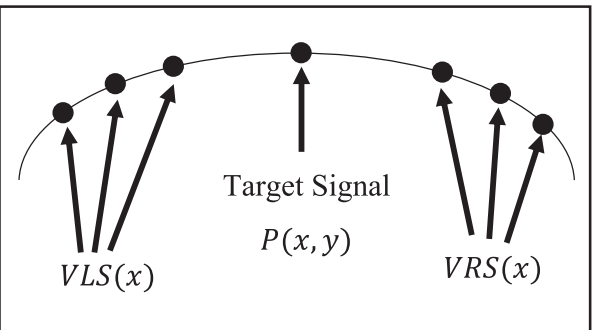

Figure 1. Target signal in Akamatsu Transform. 


$$
[D(x)]^{1 s t}=P(x)-[A(x)]^{1 s t}
$$

In previous research, the Akamatsu transformation was calculated in the x-direction. But in this research Akamatsu is also applied in the y-direction. Akamatsu transforms applied in $\mathrm{y}$-direction also divides the signal into two parts corresponding to the target signal $P(x, y)$. The section located above the $P(x, y)$ is called VTS $(y)$, which can be calculated by Eq. (10) and the part that is under the $P(x, y)$ is called $\operatorname{VBS}(y)$, which can be calculated by Eq. (11).

$$
\begin{aligned}
& \operatorname{VTS}(y)=P(x, y+1), P(x, y+2), \ldots, P(x, y+N) \\
& V B S(y)=P(x, y-1), P(x, y-2), \ldots, P(x, y-N)
\end{aligned}
$$

Similar to the x-direction Akamatsu transform, the integral value of $A(y)$ is obtained by Eq. (12).

$$
A(y)=\frac{\left[A v r S_{T}(y)+A v r S_{B}(y)\right]}{2}
$$

With $A v r S_{T}(y)$ and $A v r S_{B}(y)$ derived from Eqs (13) and (14).

$$
\begin{aligned}
& \operatorname{AvrS}_{T}(y)=\frac{S_{R}(y)}{N} \\
& \operatorname{Avr}_{B}(y)=\frac{S_{L}(y)}{N}
\end{aligned}
$$

With the first known order from Akamatsu transform then it can be continued to the next order with the above equation as many as n order, so that is obtained Eq. (15) to calculate Akamatsu transform.

$$
\begin{aligned}
& {[A(x)]^{1 s t}=A(x)} \\
& {[D(x)]^{1 s t}=P(x)-[A(x)]^{1 s t}} \\
& {[A(x)]^{\text {nth }}=A(x)} \\
& {[D(x)]^{\text {nth }}=P(x)-[A(x)]^{\text {nth }}}
\end{aligned}
$$

After obtained the integral and differential values, the new pixel value for $\mathrm{x}$-direction is obtained by Eq. (16).

$$
P_{\text {new }}(x, y)=\text { up_enh } \times D(x)+\text { down_enh } \times A(x)
$$

As for y-direction, a new pixel value is obtained by Eq. (17).

$$
P_{\text {new }}(x, y)=\text { up_enh } \times D(y)+\text { down_enh } \times A(y)
$$

where up_enh is an increasing value to increase the value of the differential waveform to obtain a clearer image. While down_enh is increasing value to decrease the integral waveform value so that the new pixel value does not exceed the 255 limit value.

\subsection{Discrete wavelet transform}

The use of wavelet transforms has been widely used in digital image processing and pattern recognition [20]. This technology also takes an important role in digital image processing. Decomposition using wavelet transform produces two-dimensional functions of time and space. Wavelet decomposition is also helpful in recognizing singularity details by analyzing the time frequencies of non-stationary signals [21-23]. Furthermore, outside there have been 
many forms of developed wavelet transforms and each has different characteristics. The discrete wavelet transform is one form of the wavelet transform, such as complex wavelet transform (CWT), Slantlet transforms (SLT) [23,24]. DWT has many good features such as multi-resolution capabilities and space-frequency localization property that makes DWT can be applied to the entire image and can be reconstructed in several image size [25]. In DWT, images are divided into 4 sub-bands: Low-Low (LL), Low-High (LH), High-Low (HL), HighHigh $(\mathrm{HH})$. The subband distribution process is carried out with two kinds of filters namely low pass and high pass filter. There are various kinds of filters that can be used such as Daubechies and Haar which are the most popular filters [26-28]. Haar filters have the advantage of being simple in structure so that they can reduce memory usage and are preferred for analyzing compact discrete signals [27]. Each sub-band holds important information about the image. LL is representative of the image and is the sub-band most similar to the original image before it is decomposed. $\mathrm{LH}, \mathrm{HL}$, and $\mathrm{HH}$ are wavelets of variations vertically, horizontally, and diagonally $[6,13,21,25]$.

Decomposition on DWT can be done using Eqs. (18) and (19) [29].

$$
\begin{gathered}
\Phi_{j, m, n}(x, y)=2^{\frac{j}{2}} \varphi\left(2^{j}-m, 2^{j}-m, 2^{j} y-n\right) \\
\Psi_{j, m, n}^{i}(x, y)=2^{\frac{j}{2}}\left(2^{j}-m, 2^{j}-m, 2^{j} y-n\right), i=\{H, V, D\}
\end{gathered}
$$

where: $i=$ wavelet $\{\mathrm{HL}, \mathrm{LH}, \mathrm{HH}\}, m, n=$ size of image

Figure 2 is a description of sub-bands decomposition proccess using in DWT using filter Haar.where $2 \downarrow 1$ : downsample columns and $1 \downarrow 2$ : downsample rows.

Figure 2 shows the decomposition process at the first level. In its development, decomposition is also done on several levels to get the most appropriate results. But in this research decomposition is only done at one level. Where later each subband will be carried out further different processes, according to the characteristics of each subband.

While reconstruction is done by inverse DWT using Eq. (20).

$$
\begin{aligned}
& f(x, y) \frac{1}{\sqrt{M N}} \sum_{m} \sum_{n} w_{\varphi}\left(j_{0}, m, n\right) \varphi_{j_{0}, m, n}(x, y) \\
& +\frac{1}{\sqrt{M N}} \sum_{i=H, V} \sum_{D j=j o}^{w} \times \sum_{m} \sum_{n} w_{\psi}^{i}\left(j_{0}, m, n\right) \Psi_{j_{0}, m, n}^{i}(x, y)
\end{aligned}
$$

\section{Proposed restoration scheme}

The proposed restoration scheme in this study is to combine DWT and Akamatsu on the input image. The input image is an image that has been given manipulations such as
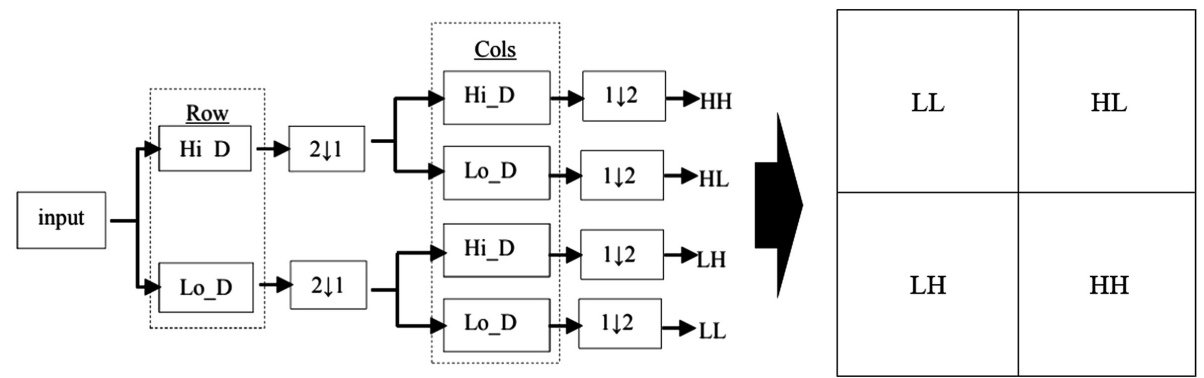

Figure 2.

Sub-bands decomposition in DWT using filter Haar.
Joint method for image restoration 
Gaussian noise, salt and pepper, and blurring. To be able to see more clearly the stages of the proposed scheme could see Figure 3.

Here is a detailed stage of the proposed restoration process in Figure 3:

1. Decompose the input image using DWT to get 4 subband LL, HL, LH, and HH.

2. Select subband HL, LH, and HH to apply Akamatsu transformation.

3. Apply y-direction Akamatsu transform on subband LH to get a new LH subband.

4. Apply x-direction Akamatsu transform on subband HL to get a new HL subband.

5. Apply two Akamatsu transforms on the HH subband. First, apply Akamatsu transform x-direction and the second Akamatsu transform y-direction. To obtain a new $\mathrm{HH}$ subband get the average value of both Akamatsu transforms with Eq. (21).

$$
H H_{\text {new }}=\frac{\left(H H_{x-\text { direction }}+H H_{y-\text { direction }}\right)}{2}
$$

6. Perform a DWT inverse to get the image of the restoration.

\section{Implementation and results}

In this study, the proposed method will be simulated using five grayscale images with $512 \times 512$ size. This image is a standard image that can be downloaded on the internet. After the image is downloaded, the image is immediately used without preprocessing. In this study, the whole trial process uses Matlab R2015a software. The image used is shown in Figure 4.

To test the proposed method, three kinds of manipulations are a blur, Gaussian noise, and salt and pepper using Matlab function. Figure 5 shows a sample that has been given manipulation.

Peak to Signal Noise Ratio (PSNR) is used to determine the degree of damage to the noised image. The value of PSNR is obtained by comparing the original image and the noised image. Equation (22) is used to calculate PSNR.

Figure 3.

The flow of Proposed Restoration Scheme.

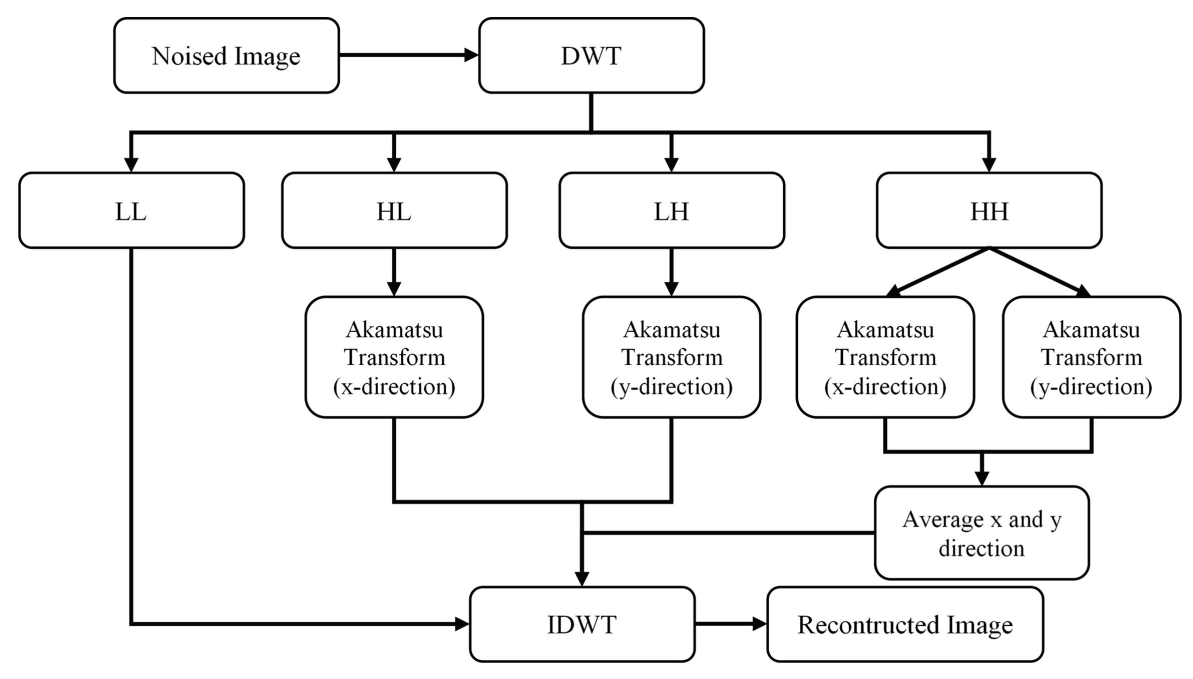




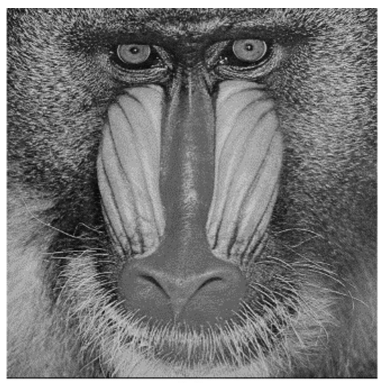

(a)

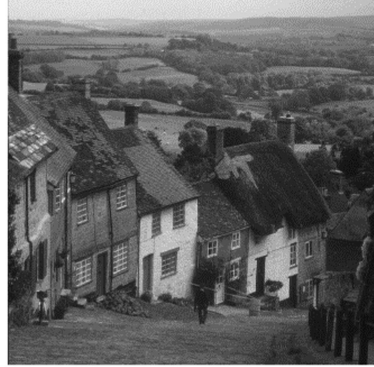

(d)

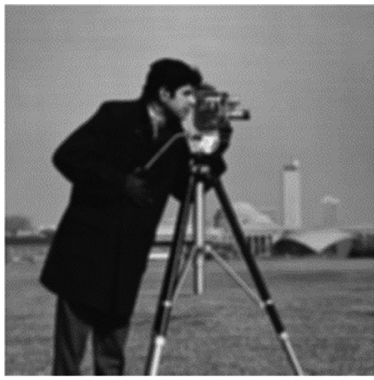

(a)

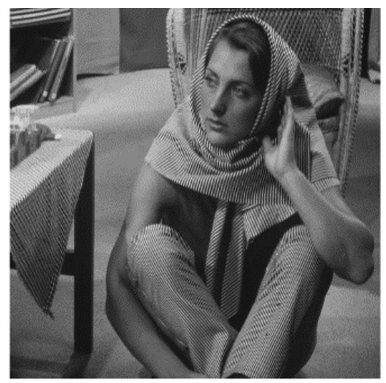

(b)

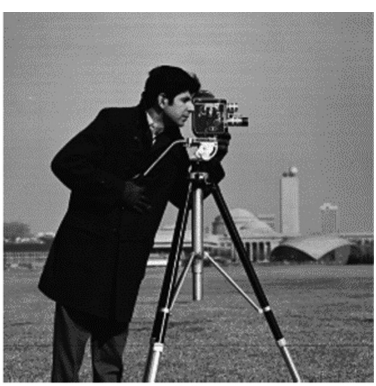

(c)

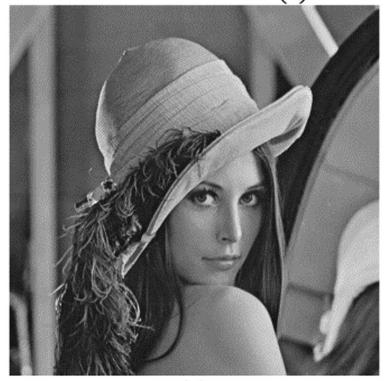

(e)

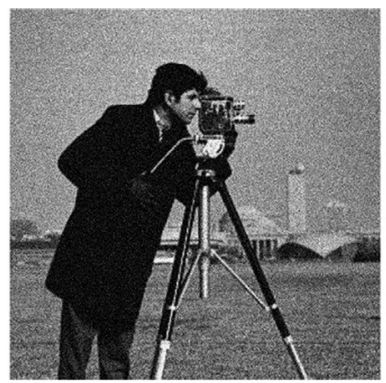

(b)

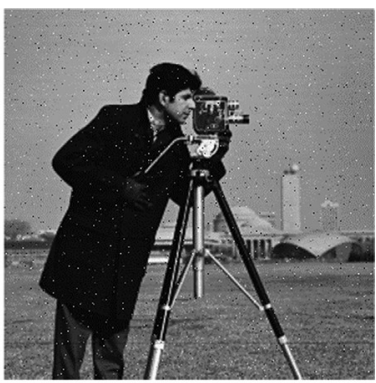

(c)
Figure 4. Original Image Used

\{(a) Babbon; (b) Barbara; (c) Cameraman; (d) Goldhill; (e) Lena\}.

Figure 5.

Noise Cameraman Image \{(a) Blur; (b) Gaussian Noise 0.01; (c) Salt and Pepper 0.01\}.

$$
P S N R=10 \log _{10} \frac{255^{2}}{\sum_{h=1}^{H-1} \sum_{g=1}^{G-1} O_{i}(h, g)-N_{i}(h, g)}
$$

where: $h, g$ is the size of the image, $O_{i}$ is an original image, and $N_{i}$ is a noised image.

Table 1 shows the PSNR value of the original image after given three kinds of noise models.

Furthermore, the restoration of the noised image with the proposed method. The proposed method combines two methods, namely wavelet transform with Haar filter and then transformed again with Akamatsu transform. Wavelet transform is performed to get four subbands, where three of the four subbands are further processed using Akamatsu transform. The processing of the Akamatsu transformation in each DWT subband is adjusted to the characteristics of each subband to optimized restoration results. Perform the 


\begin{tabular}{|c|c|c|c|}
\hline \multirow[t]{8}{*}{$\mathrm{A}$} & $\overline{\text { Image }}$ & Noise Type & PSNR \\
\hline & \multirow[t]{3}{*}{ Lena } & Gaussian & 20.0750 \\
\hline & & Salt \& Pepper & 25.3945 \\
\hline & & Blur & 27.4355 \\
\hline & \multirow[t]{3}{*}{ Barbara } & Gaussian & 20.0847 \\
\hline & & Salt \& Pepper & 25.4396 \\
\hline & & Blur & 23.5422 \\
\hline & \multirow[t]{3}{*}{ Baboon } & Gaussian & 20.0327 \\
\hline & & Salt \& Pepper & 25.6330 \\
\hline & & Blur & 20.6008 \\
\hline & \multirow[t]{3}{*}{ Cameraman } & Gaussian & 20.3898 \\
\hline & & Salt \& Pepper & 25.0744 \\
\hline & & Blur & 26.1126 \\
\hline \multirow{3}{*}{$\begin{array}{l}\text { Table } 1 . \\
\text { PSNR of the } \\
\text { noise image. }\end{array}$} & \multirow[t]{3}{*}{ Goldhill } & Gaussian & 20.1134 \\
\hline & & Salt \& Pepper & 25.2362 \\
\hline & & Blur & 26.7294 \\
\hline
\end{tabular}

Akamatsu y-direction transform on the LH subband, the x-direction on the HL subband and the xy-direction on the HH subband. This is done because the LH subband has a middle frequency horizontally, $\mathrm{HL}$ has a vertically middle frequency and $\mathrm{HH}$ has a diagonal high frequency $[6,13,21,25]$. Table 2 shows the results and PSNR values of the reconstructed images from the proposed restoration method.

\section{Comparative and analysis}

To evaluate the performance of the proposed method, the restoration of the proposed method were compared with the results of method restoration on the research of Karungaru et al. [5] and Megha et al. [6]. In this research, both methods have been replicated to compare the results of image restoration. This is done because the data set of images used are different, so it needs to be replicated so that it can be compared with the same image dataset. Comparative measurements were made with PSNR shown in Table 3.

As can be seen in Table 3 , the PSNR value of the proposed method of restoration appears better than the two previous methods. Visually the difference of image result of restoration can be seen in Figures 6-8.

Based on the PSNR values contained in Table 3, proves that the results of image restoration by the proposed method appear superior to the previous method. Visually, Figures 6-8 also show that the proposed method appears to be superior. Only, in the blur, the results of restoration by the method [5] appear more clear and sharp when compared with the proposed method. However, the result of restoration on the method [6] creates new noise in the form of lines on the edge of the image, as shown in Figure 6(a).

\section{Conclusions}

Based on the results of the analysis and comparison, it can be concluded that the proposed method has better restoration compared to the method [5] and method [6] in all types of manipulations. The restoration of Gaussian noise and salt and pepper manipulations also reduce and smooth the noise generated. While the restoration using the method [5] even causes a blur effect. This happens because the Akamatsu transformation has characteristics that can change the image information by changing the differential value with the down_enh value. While the proposed method applies changes to this information on sub-band HL, LH, 


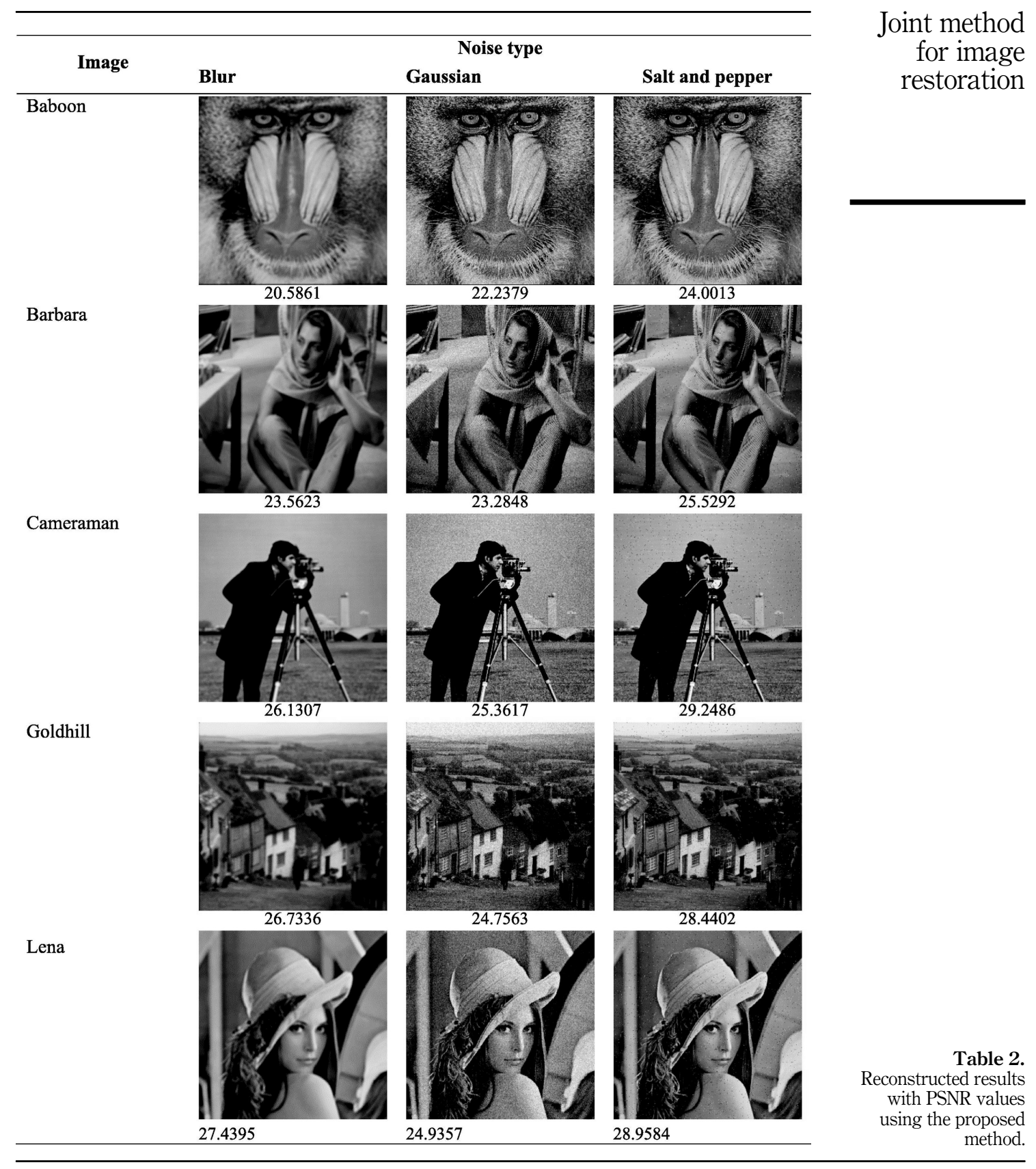




\section{$\mathrm{ACI}$}

\begin{tabular}{llccc}
\hline Image & Noise type & Proposed method & The method in [5] & The method in [6] \\
\hline Lena & Gaussian & 24.9357 & 23.4659 & 17.0212 \\
& Salt \& Pepper & 28.9584 & 25.9581 & 19.0746 \\
Barbara & Blur & 27.4395 & 26.3382 & 19.2786 \\
& Gaussian & 23.2848 & 23.0617 & 18.0083 \\
& Salt \& Pepper & 25.5292 & 25.1769 & 20.3796 \\
Baboon & Blur & 23.5623 & 23.0914 & 19.6397 \\
& Gaussian & 22.2379 & 22.1477 & 17.1517 \\
& Salt \& Pepper & 24.0013 & 23.8401 & 19.2273 \\
Cameraman & Blur & 20.5861 & 20.4113 & 17.5664 \\
& Gaussian & 25.3617 & 22.8571 & 17.2854 \\
Goldhill & Salt \& Pepper & 29.2486 & 24.7714 & 18.6267 \\
& Blur & 26.1307 & 25.3826 & 18.8358 \\
& Gaussian & 24.7563 & 23.2829 & 17.1602 \\
& Salt \& Pepper & 28.4402 & 25.4686 & 18.7861 \\
& Blur & 26.7336 & 25.6285 & 18.6463 \\
\hline
\end{tabular}

Figure 6.

Reconstructed

Cameraman Image

from Blur

manipulations

\{(a) Method in [5];

(b) Method in [6];

(c) Proposed Method\} .

Figure 7.

Reconstructed

Cameraman Image

from Gaussian Noise manipulations

\{(a) Method in [5];

(b) Method in [6];

(c) Proposed Method\}.

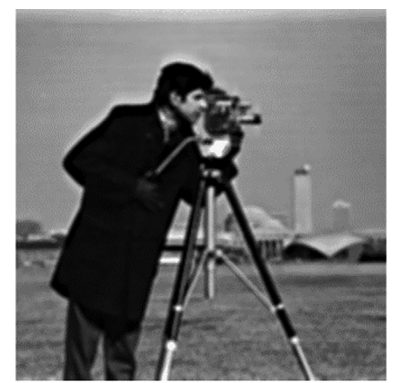

(a)

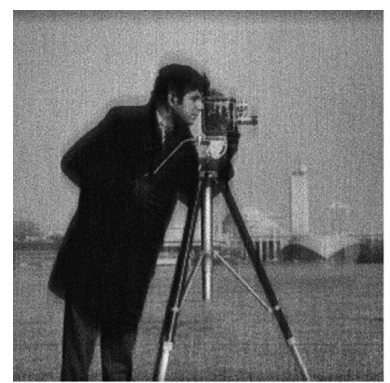

(a)

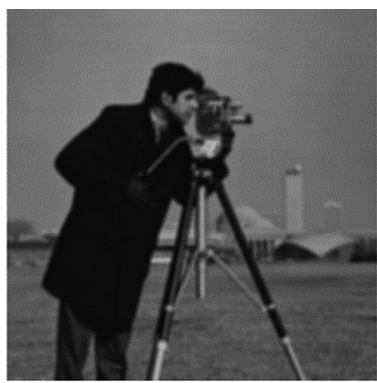

(b)

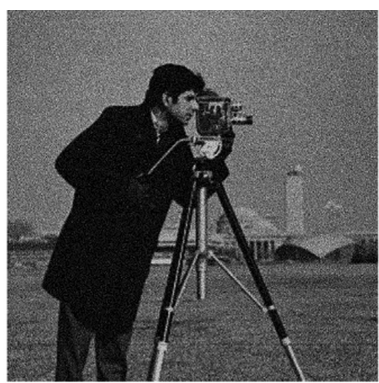

(b)

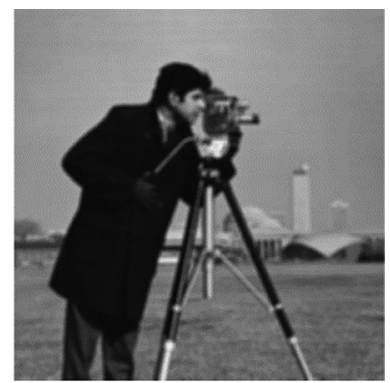

(c)

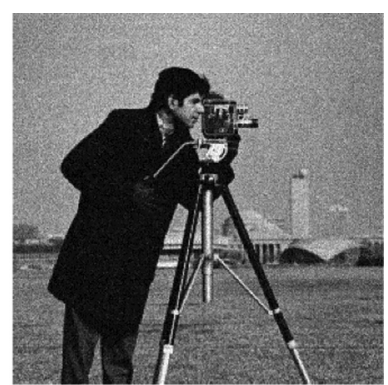

(c)

and $\mathrm{HH}$. So after the inverse DWT, the original image characteristics did not change significantly but can eliminate the noise that occurs in the image. Method [6] has a different character, although noise is reduced, the energy in the image of the restoration in the image is also reduced so that the image appears darker and the resulting PSNR value is less good. So it can be concluded that the proposed method can work better on various manipulations and can maintain the value of image approximation. In future studies, several wavelet filters can 


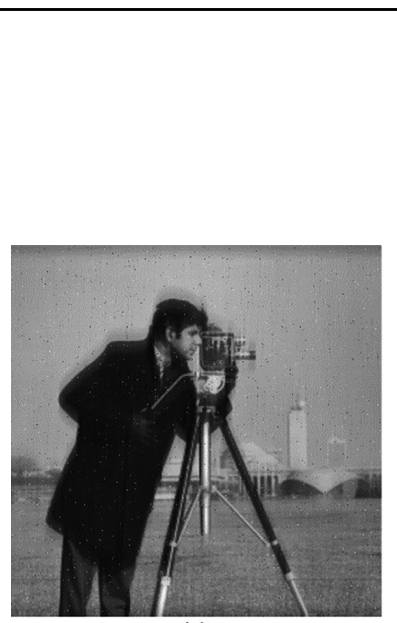

(a)

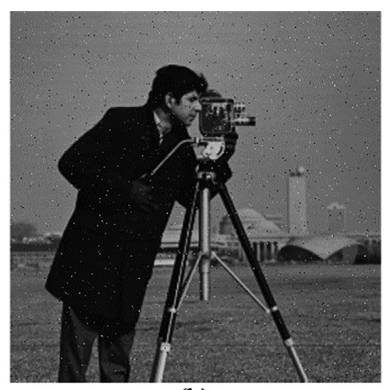

(b)

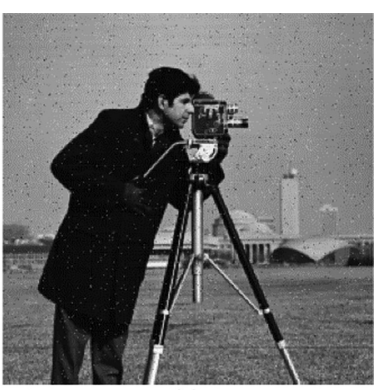

(c)
Joint method for image restoration

Figure 8.

Reconstructed Cameraman Image from Salt and Peppers manipulations $\{(\mathrm{a})$

Method in [5]; (b)

Method in [6]; (c) Proposed Method\}.

be used, as well as improved wavelet transforms and wavelet transform levels to analyze the results so that more optimal restoration is obtained. The use of other transformations can also be combined to get better image restoration.

\section{References}

[1] C. Solomon, T. Breckon, Fundamentals of Digital Image Processing, 2011. doi: 10.1007/978-981-107272-7_1.

[2] K. Kaibiche, S. Saadi, D. Chikouche, Z. Messali, Restoration of stained old manuscripts via a hybrid wavelet and bilateral filtering system, J. King Saud Univ. - Comput Inf. Sci. (2017) 493-498, https://doi.org/10.1016/j.jksuci.2016.09.003, 29.

[3] P. Singh, R. Shree, M. Diwakar, A new SAR image despeckling using correlation based fusion and method noise thresholding, J. King Saud Univ. - Comput Inf. Sci. (2018), https://doi.org/10.1016/j. jksuci.2018.03.009.

[4] B. Langari, S. Vaseghi, A. Prochazka, B. Vaziri, F. Tahmasebi Aria, Edge-guided image gap interpolation using multi-scale transformation, IEEE Trans. Image Process. 25 (2016) 4394-4405, https://doi.org/10.1109/TIP.2016.2590825.

[5] S. Karungaru, M. Sugizaki, M. Fukumi, Y. Mitsukura, N. Akamatsu, Out-of-focus blur image restoration using the Akamatsu transform, in: 2009 35th Annu. Conf. IEEE Ind. Electron, IEEE (2009) 4257-4261, https://doi.org/10.1109/IECON.2009.5415072.

[6] P. Megha, M. Swarna, V. Sowmya, K.P. Soman, Low contrast satellite image restoration based on adaptive histogram equalization and discrete wavelet transform, in: 2016 Int. Conf. Commun. Signal Process., IEEE, 2016: pp. 0402-0406. doi: 10.1109/ICCSP.2016.7754166.

[7] Y. Jiang, Y.P. Peng, J. Wang, Study on algorithm of image restoration based on stochastic resonance and wavelet transforming, Sixth Int. Conf. Instrum. Meas. Comput Commun. Control, IEEE 2016 (2016) 416-421, https://doi.org/10.1109/IMCCC.2016.137.

[8] E. Hostalkova, O. Vysata, A. Prochazka, Multi-Dimensional Biomedical Image De-Noising using Haar Transform, in: 2007 15th Int. Conf. Digit. Signal Process, IEEE (2007) 175-178, https://doi. org/10.1109/ICDSP.2007.4288547.

[9] R. Jenifa, T. Latha, C.H. Sulochana, Image restoration using modified Anscombe transform and non linear multiresolution median transform, in: 2015 Glob. Conf. Commun. Technol, IEEE (2015) 139-144, https://doi.org/10.1109/GCCT.2015.7342640. 
[10] R. Dhannawat, A.B. Patankar, Improvement to blind image denoising by using local pixel grouping with SVD, Procedia Comput. Sci. 79 (2016) 314-320, https://doi.org/10.1016/J.PROCS. 2016.03.041.

[11] M. Manchanda, D. Gambhir, Channel noise reduction for compressed images using singular value decomposition, in: 2014 5th Int. Conf. - Conflu. Next Gener. Inf. Technol. Summit, IEEE (2014) 613-618, https://doi.org/10.1109/CONFLUENCE.2014.6949241.

[12] S. Suresh, D. Das, S. Lal, D. Gupta, Image quality restoration framework for contrast enhancement of satellite remote sensing images, Remote Sens. Appl. Soc. Environ. 10 (2018) 104119, https://doi.org/10.1016/j.rsase.2018.03.008.

[13] A. Setyono, D.R.I.M. Setiadi, Image watermarking using discrete wavelet-tchebichef transform, Indones. J. Electr. Eng. Comput. Sci. 16 (2019) 1416-1423, https://doi.org/10.11591/ijeecs.v16.i3.

[14] A. Naz, A. Talukdar, K.K. Sarma, Digital Image Restoration Using Discrete Wavelet Transform Based Approach, IRNet Trans. Electr. Electron. Eng. 1 (2012) 53-57 (accessed 11.06.19) https:// www.academia.edu/2439876/DIGITAL_IMAGE_RESTORATION_USING_DISCRETE_ WAVELET_TRANSFORM_BASED_APPROACH.

[15] R.A. Alotaibi, L.A. Elrefaei, Text-image watermarking based on integer wavelet transform (IWT) and discrete cosine transform (DCT), Appl. Comput. Informat. (2018), https://doi.org/10.1016/J. ACI.2018.06.003.

[16] A. Vaish, S. Gautam, M. Kumar, A wavelet based approach for simultaneous compression and encryption of fused images, J. King Saud Univ. - Comput Inf. Sci. 31 (2019) 208-217, https://doi. org/10.1016/J.JKSUCI.2017.01.005.

[17] A. Setyono, D.R.I.M. Setiadi, M. Muljono, StegoCrypt method using wavelet transform and onetime pad for secret image delivery, in: 2017 4th Int. Conf. Inf. Technol. Comput. Electr. Eng., IEEE (2017) 203-207, https://doi.org/10.1109/ICITACEE.2017.8257703.

[18] S.M. Mahbubur Rahman, M.O. Ahmad, M.N.S. Swamy, Improved image restoration using wavelet-based denoising and fourier-based deconvolution, in: 2008 51st Midwest Symp. Circuits Syst, IEEE (2008) 249-252, https://doi.org/10.1109/MWSCAS.2008.4616783.

[19] S. Karungaru, T. Kamei, M. Fujiwara, N. Akamatsu, Vowel recognition using akamatsu integral and differential transforms and differential transforms, Int. J. Adv. Intell. 1 (2014) 125-140.

[20] M.A. Muqeet, R.S. Holambe, Local binary patterns based on directional wavelet transform for expression and pose-invariant face recognition, Appl. Comput. Informat. (2017), https://doi.org/10. 1016/J.ACI.2017.11.002.

[21] A. Sharma, A. Khunteta, Satellite image enhancement using discrete wavelet transform, singular value decomposition and its noise performance analysis, in: 2016 Int. Conf. Micro-Electronics Telecommun. Eng., IEEE (2016) 594-599, https://doi.org/10.1109/ICMETE.2016.32.

[22] A. Prochazka, I. Sindelarova, J. Ptacek, Image de-noising and restoration using wavelet transform, in: 2003 Eur. Control Conf, IEEE (2003) 382-386, https://doi.org/10.23919/ECC.2003.7084984.

[23] I.W. Selesnick, R.G. Baraniuk, N.G. Kingsbury, The Dual-Tree Complex Wavelet Transform, IEEE Signal Proc Mag. (2005) 123-151, accessed 30.08.19.

[24] I.W. Selesnick, The Slantlet Transform, IEEE Trans. Signal Process. 47 (1999) (accessed 31.08.19.) https://pdfs.semanticscholar.org/7749/e57ff679a7046c9ceb18080789b060761cf8.pdf.

[25] S. Trambadia, H. Mayatra, Image inpainting based on Discrete Wavelet Transform (DWT) technique, in: 2016 Online Int. Conf. Green Eng. Technol, IEEE (2016) 1-6, https://doi.org/10.1109/ GET.2016.7916794.

[26] F.M. Bayer, A.J. Kozakevicius, R.J. Cintra, An iterative wavelet threshold for signal denoising, Sign. Process. 162 (2019) 10-20, https://doi.org/10.1016/j.sigpro.2019.04.005.

[27] M. Schimmack, S. Nguyen, P. Mercorelli, Anatomy of haar wavelet filter and its implementation for signal processing, IFAC-PapersOnLine. 49 (2016) 99-104, https://doi.org/10.1016/J.IFACOL. 2016.07.160. 
[28] Y. Liu, S. Tang, R. Liu, L. Zhang, Z. Ma, Secure and robust digital image watermarking scheme using logistic and RSA encryption, Expert Syst. Appl. 97 (2018) 95-105, https://doi.org/10.1016/J. ESWA.2017.12.003.

[29] Z. Ye, H. Mohamadian, Y. Ye, Information Measures for Biometric Identification via 2D Discrete Wavelet Transform, in: 2007 IEEE Int. Conf. Autom. Sci. Eng, IEEE (2007) 835-840, https://doi. org/10.1109/COASE.2007.4341670.

\section{Corresponding author}

De Rosal Ignatius Moses Setiadi can be contacted at: moses@dsn.dinus.ac.id

For instructions on how to order reprints of this article, please visit our website:

www.emeraldgrouppublishing.com/licensing/reprints.htm

Or contact us for further details: permissions@emeraldinsight.com 